\title{
Characterization of one- and two-dimensional switch lines for controlling traveling pulses
}

\author{
Koichi Narahara ${ }^{a}$ \\ Graduate School of Science and Engineering, Yamagata University, 4-3-16 Jonan, \\ Yonezawa, Yamagata 992-8510, Japan \\ a)narahara@yz.yamagata-u.ac.jp
}

\begin{abstract}
We characterize the properties of one- and two-dimensional transmission lines periodically loaded with electronic switches, for use in managing amplitude and propagation orientations of pulses traveling on the lines. The detection of the input voltage level is successfully demonstrated experimentally using the test switch line. Moreover, the orientation of pulse propagation is controllable in a twodimensional switch line.
\end{abstract}

Keywords: nonlinear waves, tunneling diodes, switch lines

Classification: Science and engineering for electronics

\section{References}

[1] I. Richer, "The switch line: a simple lumped transmission line that can support unattenuated propagation," IEEE Trans. Circuit Theory, vol. 13, no. 4, pp. 388-392, Dec. 1966.

[2] K. Narahara and A. Yokota, "Experimental characterization of short pulse generation in switch lines," IEICE Electron. Express, vol. 5, no. 22, pp. 973-977, Nov. 2008.

[3] H. Sugiyama, H. Matsuzaki, Y. Oda, H. Yokohama, T. Enoki, and T. Kobayashi, "Metal-organic vapor-phase epitaxy of InP-based resonant tunneling diodes with a strained $\mathrm{In}_{0.8} \mathrm{Ga}_{0.2}$ As well and AlAs barriers," Jpn. J. Appl. Phys., vol. 44, no. 10, pp. 7314-7318, Oct. 2005.

\section{Introduction}

A switch line was originally defined as a lumped transmission line containing a series resistor, shunt capacitor, and shunt switch in each section (the switch is open for voltages greater than some fixed threshold and closed otherwise) [1]. We added a series inductor to create a switch line for use in high-speed electronics, investigated the quasi-steady propagation of a pulse on a onedimensional switch line, and reported that the pulse traveling on the line becomes greatly compressed [2]. The resulting short pulse can be applied to high-resolution measurement and high-speed communication systems. 
The switch line is useful for more than just short-pulse generation. Its potential is widened by considering two-dimensional lines. This article discusses two such switch line applications: the detection of pulse amplitudes on a one-dimensional switch line and the control of a pulse's propagation orientation on a two-dimensional switch line.

\section{Operating principle}

Figures 1 (a) and (b) show unit cells of one- and two-dimensional switch lines, respectively. $L, C$, and $I_{s w}$ represent the series inductor, shunt capacitor and current in the shunt switch, respectively. The ideal current-voltage relationship is shown in Fig. 1(c). The switch opens for voltages greater than a threshold $V_{t h}$ and exhibits a constant conductance $G_{0}$ for voltages less than $V_{t h}$. Tunneling diodes could successfully simulate the switches by taking their peak voltage as $V_{t h}$. We refer to the voltage below $V_{t h}$ as region I and that greater than $V_{t h}$ as region II. We consider only pulses that are greater than $V_{t h}$; therefore, the pulse is influenced by finite shunt conductance in region
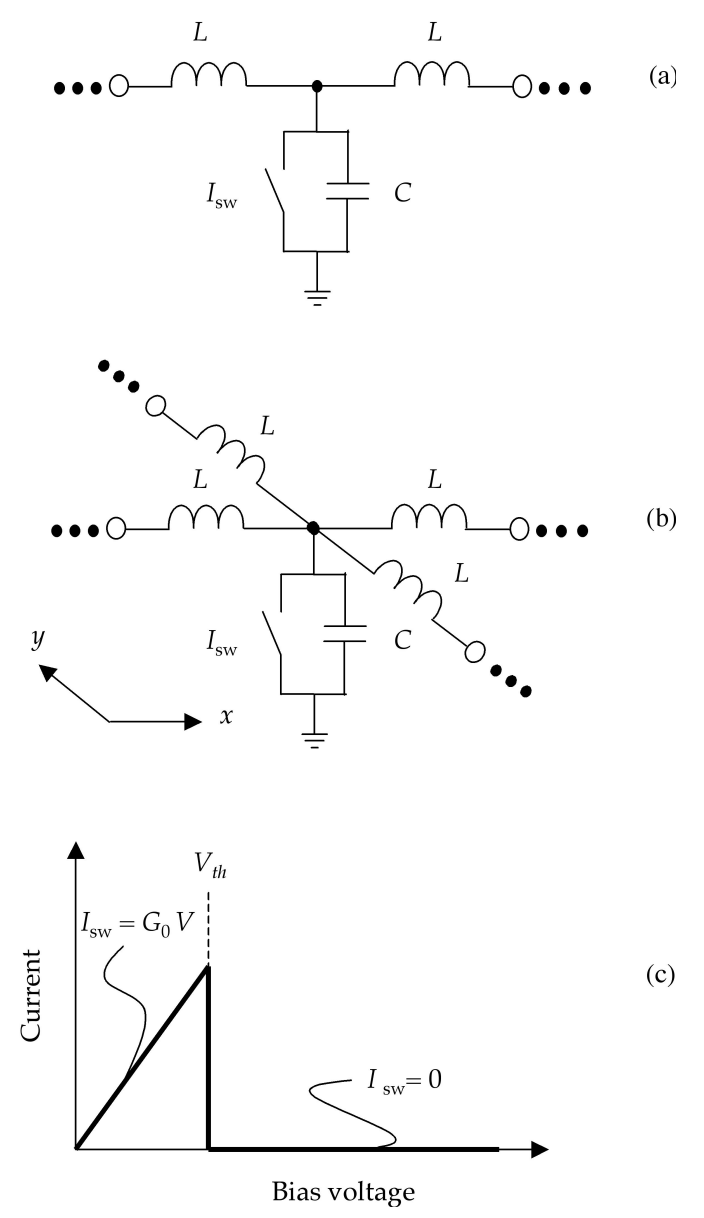

Fig. 1. Equivalent representation of switch lines. (a) A one-dimensional switch line, (b) a twodimensional switch line, and (c) a model of the shunt switches. $L$ is the series inductance and $C$ is the shunt capacitance. The switch has a unique threshold $V_{t h}$. 


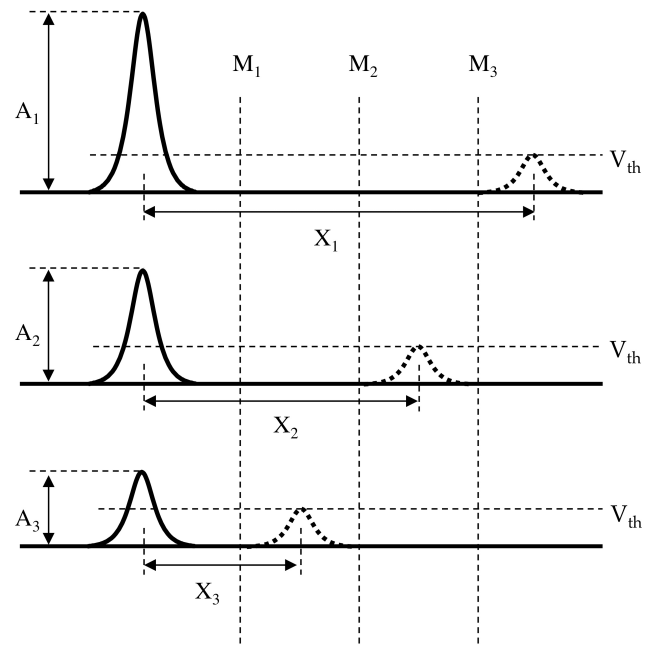

Fig. 2. Detection of input voltage levels using a onedimensional switch line. The larger the amplitude of the input pulse, the longer the pulse travels on the switch line. We can detect every pulse at $M_{1}$, but only the largest pulse at $M_{3}$.

I and is completely loss-free in region II; therefore, the pulse is attenuated partially at the bottom. It is then expected that a pulse of large amplitude can travel on the line longer than one of small amplitude (Fig. 2).

Pulses with amplitude $A_{1}, A_{2}$, and $A_{3}\left(A_{1}>A_{2}>A_{3}\right)$ travel on the switch line by $X_{1}, X_{2}$, and $X_{3}\left(X_{1}>X_{2}>X_{3}\right)$, respectively. When we monitor the waveform at $M_{1}$, we detect the pulse regardless of the amplitude. However, because the smallest pulse disappears before it reaches $M_{2}$, we can detect only those pulses of amplitude of $A_{1}$ and $A_{2}$. Similarly, at $M_{3}$, we can detect only the largest pulse. By specifying the monitoring cell on the switch line, we can set a threshold voltage level to detect pulses as needed. The next section describes the experimental demonstration of this input-level decision. When several points are excited by the voltage pulses in a two-dimensional switch line, the propagating pulses overlap and gain amplitude. As mentioned above, the larger pulse survives longer than the smaller pulses; therefore, as time progresses, the overlapped pulses have finite amplitude. The simplest application of this phenomena is to control a pulse's propagation orientation by the arrangement of cells where the pulses are applied. To examine the validity of this application, we numerically solved the transmission-line equations of a two-dimensional switch line. We set $C, L$, $V_{t h}$, and $G_{0}$ as $1.0 \mathrm{pF}, 1.0 \mathrm{nH}, 0.1 \mathrm{~V}$, and $0.04 \mathrm{~S}$, respectively. The total cell size was $100 \times 100$. Each edge cell was terminated using a $50-\Omega$ resistor. An impulse having a Gaussian form with amplitude $1.0 \mathrm{~V}$ and full-width at half-maximum (FWHM) $100.0 \mathrm{ps}$ was input at cells located at $(45,45)$ and $(55,55)$.

Figure 3 shows the numerical results of pulse propagation. Waveforms at times $0.4,0.8,1.2$, and $1.6 \mathrm{~ns}$ passed after the pulse incidence are shown in Fig. 3 (a), (b), (c), and (d), respectively. Each pulse starts isotropic propa- 

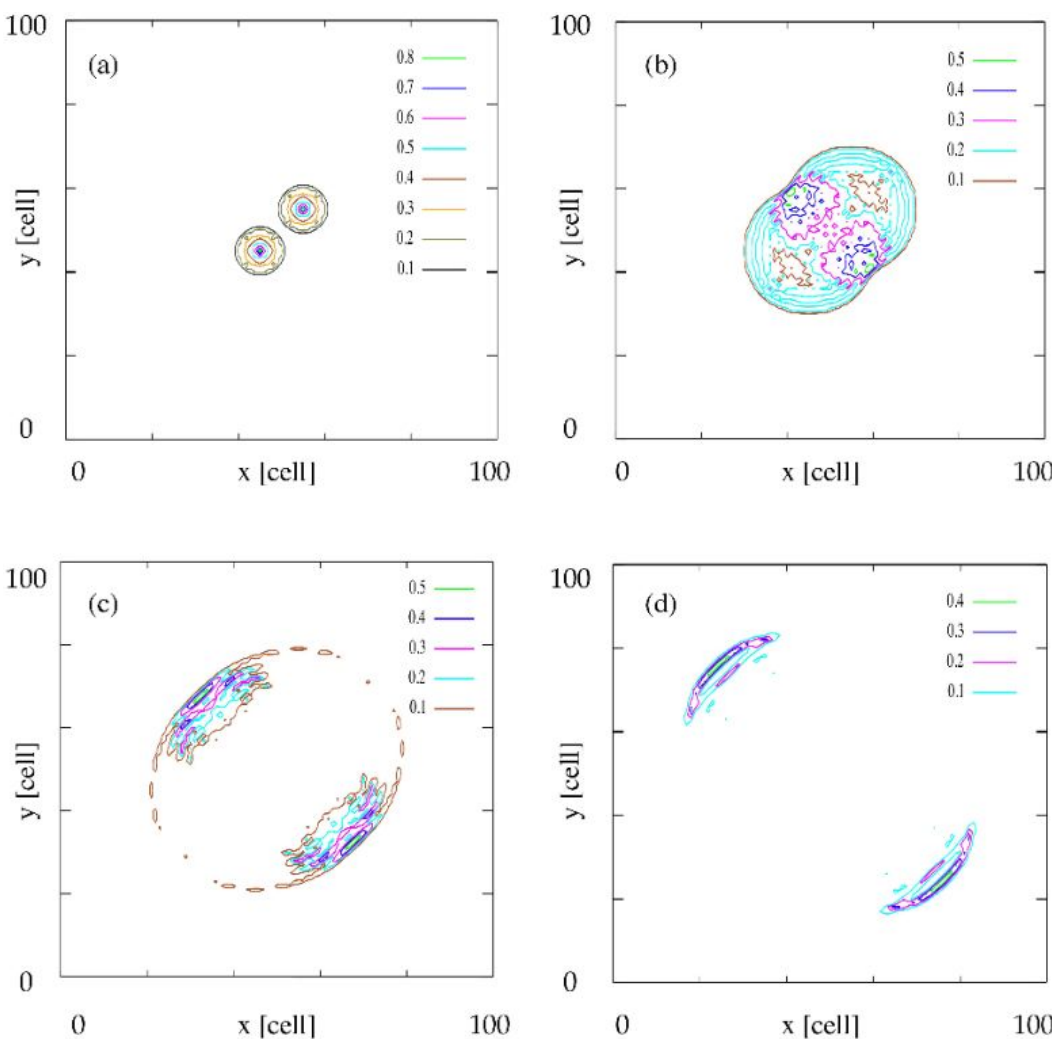

Fig. 3. Pulse propagation in two-dimensional switch lines. Two cells located at $(45,45)$ and $(55,55)$ are excited by a Gaussian pulse. The waveforms at times (a) 0.4 , (b) 0.8 , (c) 1.2 , and (d) $1.6 \mathrm{~ns}$ passed after the pulse incidence.

gation and overlaps the other pulses as observed in Fig. 3(b). The parts of the overlapped pulses of large amplitude survive, as shown in Fig. 3 (c). The remainders of the pulses on the bisection of the line connecting two excitation cells are shown in Fig. 3 (d). By changing the positions of the two excitation cells, we can control the propagation orientation of the surviving pulses as needed.

\section{Experiments}

We used a 50-section one-dimensional switch line whose unit section is shown in Fig. 1 (a). The circuit is built on a standard bread board. The shunt electronic switches are NEC 1S1763 Esaki diodes. The peak current and voltage, which corresponds to $V_{t h}$ in the ideal switch model, are typically $6.0 \mathrm{~mA}$ and $60 \mathrm{mV}$, respectively. The typical parasitic capacitance is $30.0 \mathrm{pF}$. Series inductances and shunt capacitances are implemented using $1.0-\mu \mathrm{H}$ inductors (TDK SP0508) and capacitors (TDK FK24C0G1), respectively. The design parameters are estimated as $L=1.0 \mu \mathrm{H}$, and $G_{0}=0.1 \mathrm{~S}$. The test switch line is fed by a pulse signal generated by an Agilent $81150 \mathrm{~A}$ function generator. A Gaussian pulse with an FWHM of $1.0 \mu \mathrm{s}$ is input. The signals along the test switch line are detected and monitored in the time domain using an Agilent DSO90254A oscilloscope. 

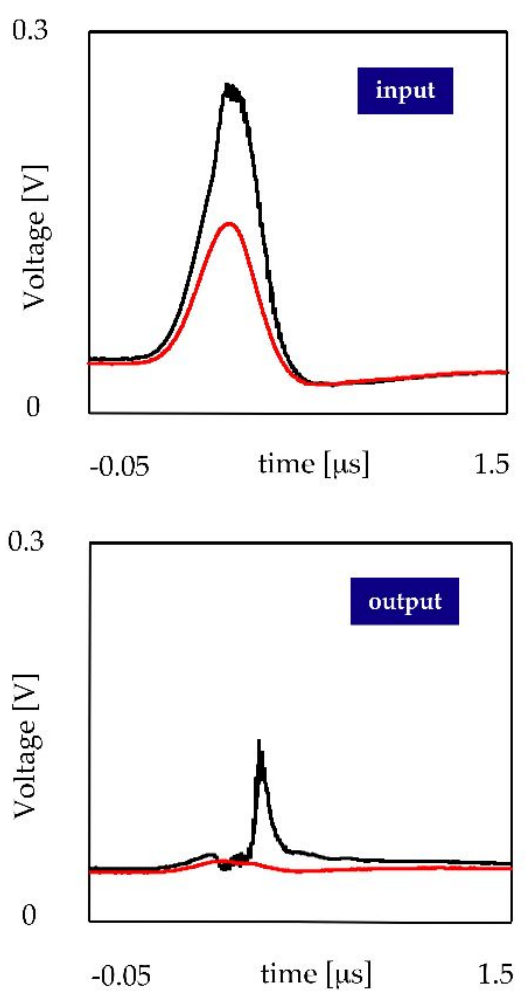

(b)

Fig. 4. Detection of input pulse amplitude. (a) The waveforms monitored at $n=1$ and (b) those monitored at $n=5$.

Figure 4 shows the measured results. The waveforms were monitored at a fixed cell for two pulses having different amplitudes. By arranging the input amplitudes, only the larger pulse can be detected. Figures 4 (a) and (b) show the voltage waveforms monitored at the cells $n=1$ and 5 , respectively. The blue waveforms in Figs. 4 (a) and (b) are for the pulse of amplitude $720 \mathrm{mV}$; the red waveforms are for the pulse of amplitude $490 \mathrm{mV}$. Only the blue pulse is detected at the output. It is thus established that only the larger pulse is detected at the output.

\section{Conclusion}

We introduced two pulse-management schemes using switch lines, and demonstrated that they can be used to manage pulse amplitude and propagation orientation. Our approach could be scaled from its current $\mathrm{MHz}$ form into microwave, millimeter-wave, and terahertz forms, for implementation with state-of-the-art tunneling devices such as InP RTDs [3]. 